\title{
Correction to: Effects of endocrine disruptors on fetal testis development, male puberty, and transition age
}

\author{
Francesco Cargnelutti ${ }^{1}$ - Andrea Di Nisio ${ }^{2}$ Francesco Pallotti ${ }^{1} \cdot$ Iva Sabovic $^{2}$ - Matteo Spaziani ${ }^{3}$. \\ Maria Grazia Tarsitano ${ }^{3}$. Donatella Paoli $\mathbb{D}^{1} \cdot$ Carlo Foresta $^{2}$
}

Published online: 4 January 2021

(c) Springer Science+Business Media, LLC, part of Springer Nature 2021

Correction to: Endocrine

https://doi.org/10.1007/s12020-020-02436-9

In the original publication, a reference was mistakenly omitted during manuscript preparation. The authors added a missing citation.

Authors ref number has been changed in table 3, 4 and 5 .

The corrected tables are given below.
Citation error in the article has been corrected.

The original article has been corrected.

\section{Reference}

57. C.L. Schwartz, S. Christiansen, A.M. Vinggaard, M. Axelstad, U. Hass, T. Svingen, Anogenital distance as a toxicological or clinical marker for fetal androgen action and risk for reproductive disorders. Arch. Toxicol. 93(2), 253-272 (2019)
The original article can be found online at https://doi.org/10.1007/ s12020-020-02436-9.

Donatella Paoli

donatella.paoli@uniroma1.it

1 Laboratory of Seminology-Sperm Bank "Loredana Gandini”, Department of Experimental Medicine, "Sapienza" University of Rome, Viale del Policlinico 155, 00161 Rome, Italy

2 Department of Medicine, Operative Unit of Andrology and Medicine of Human Reproduction, University of Padova, Via Giustiniani, 2, 35128 Padua, Italy

3 Department of Experimental Medicine, "Sapienza" University of Rome, Viale del Policlinico 155, 00161 Rome, Italy 


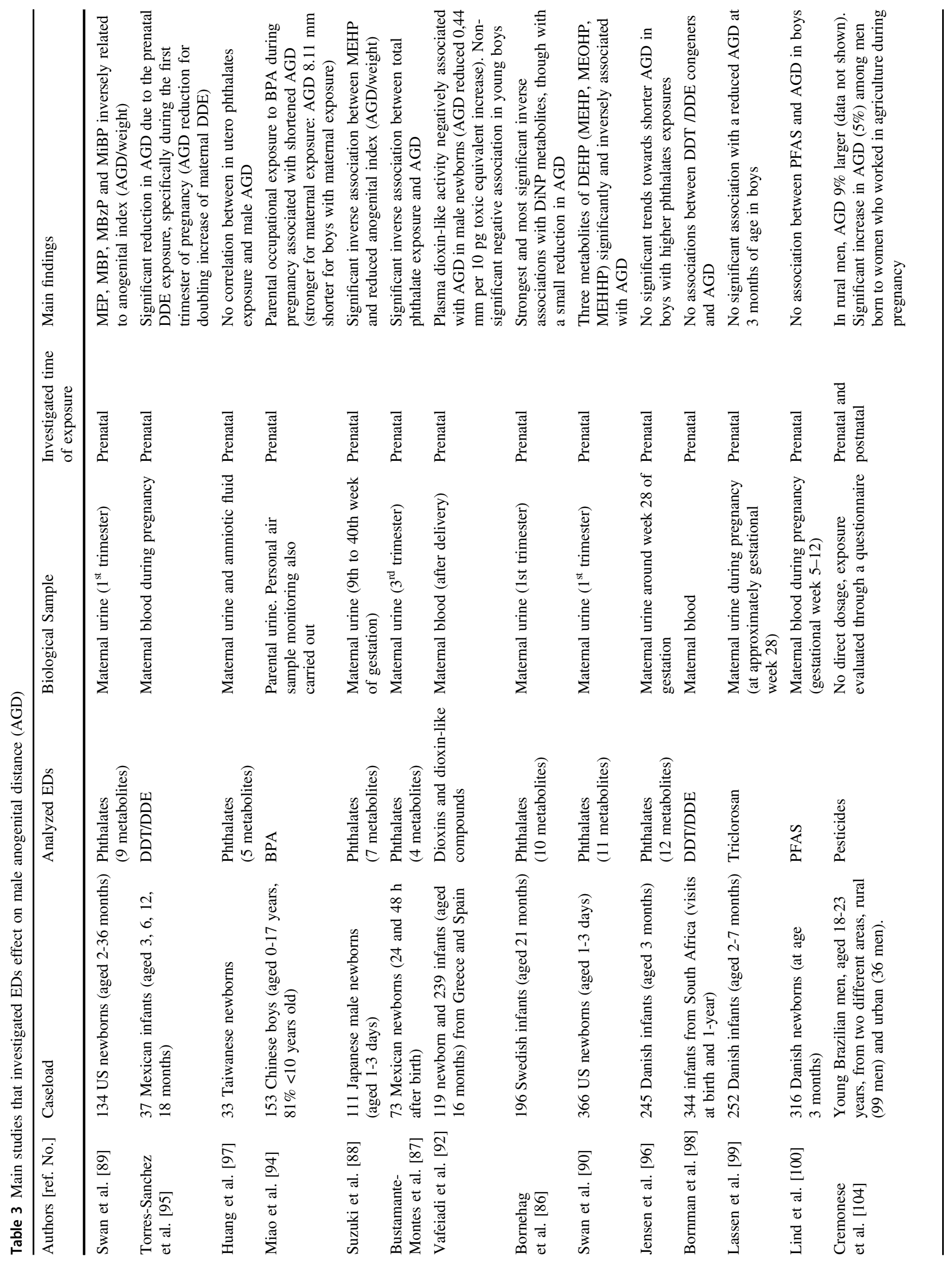




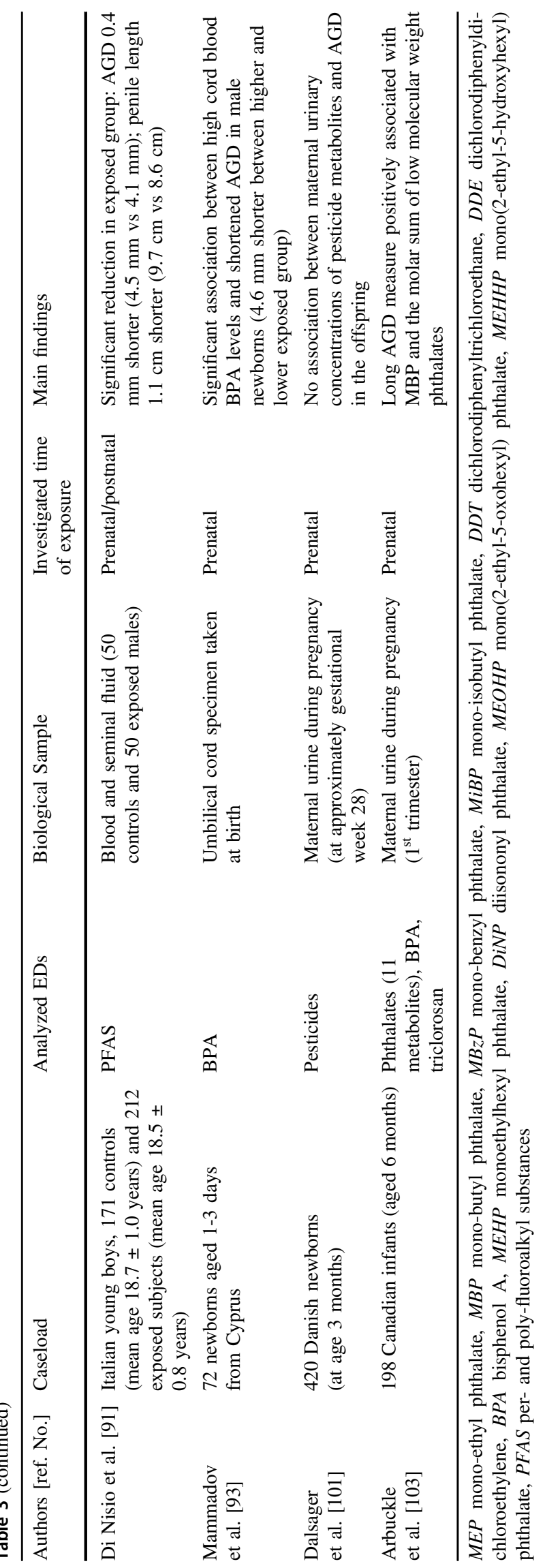




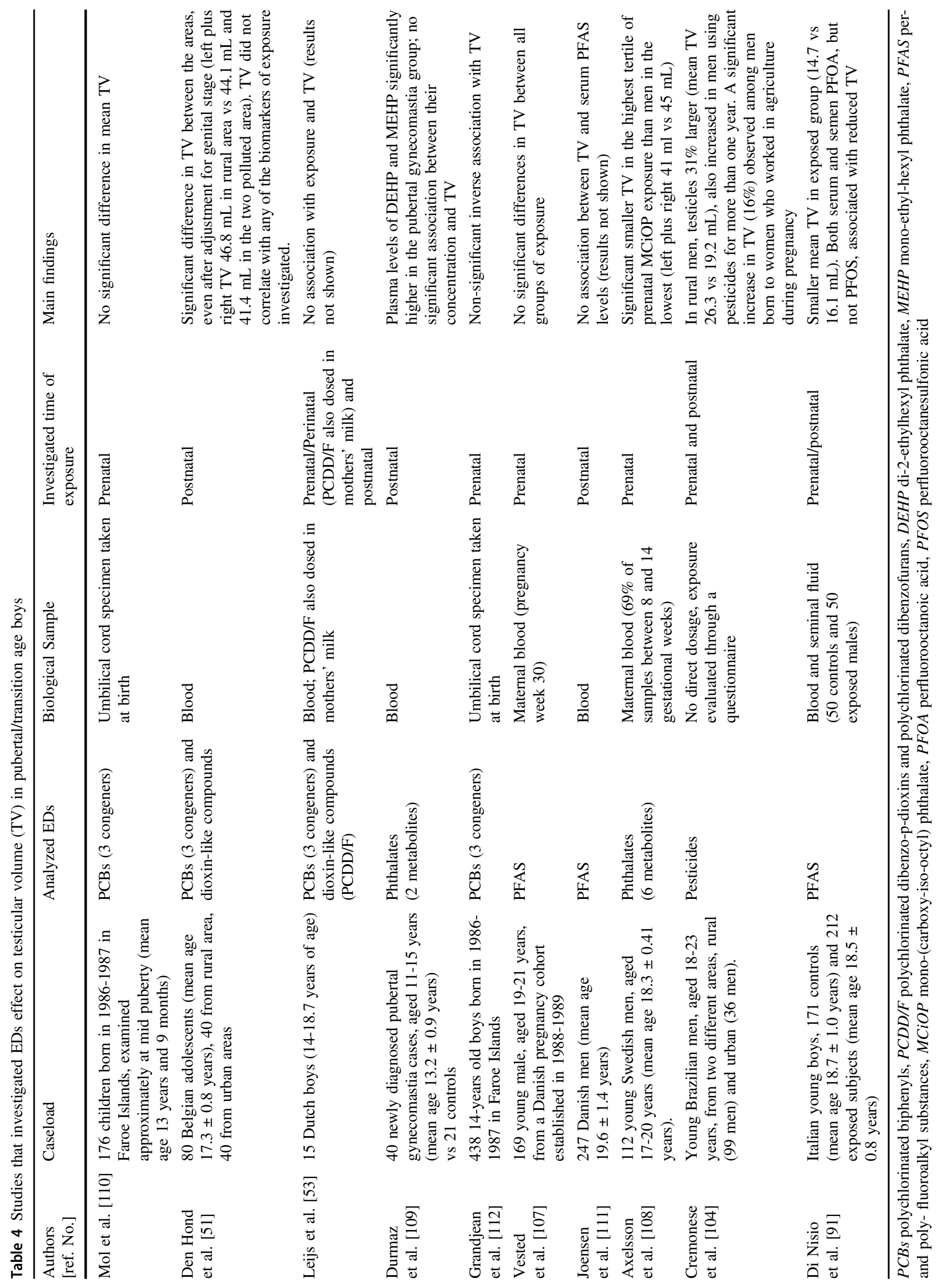




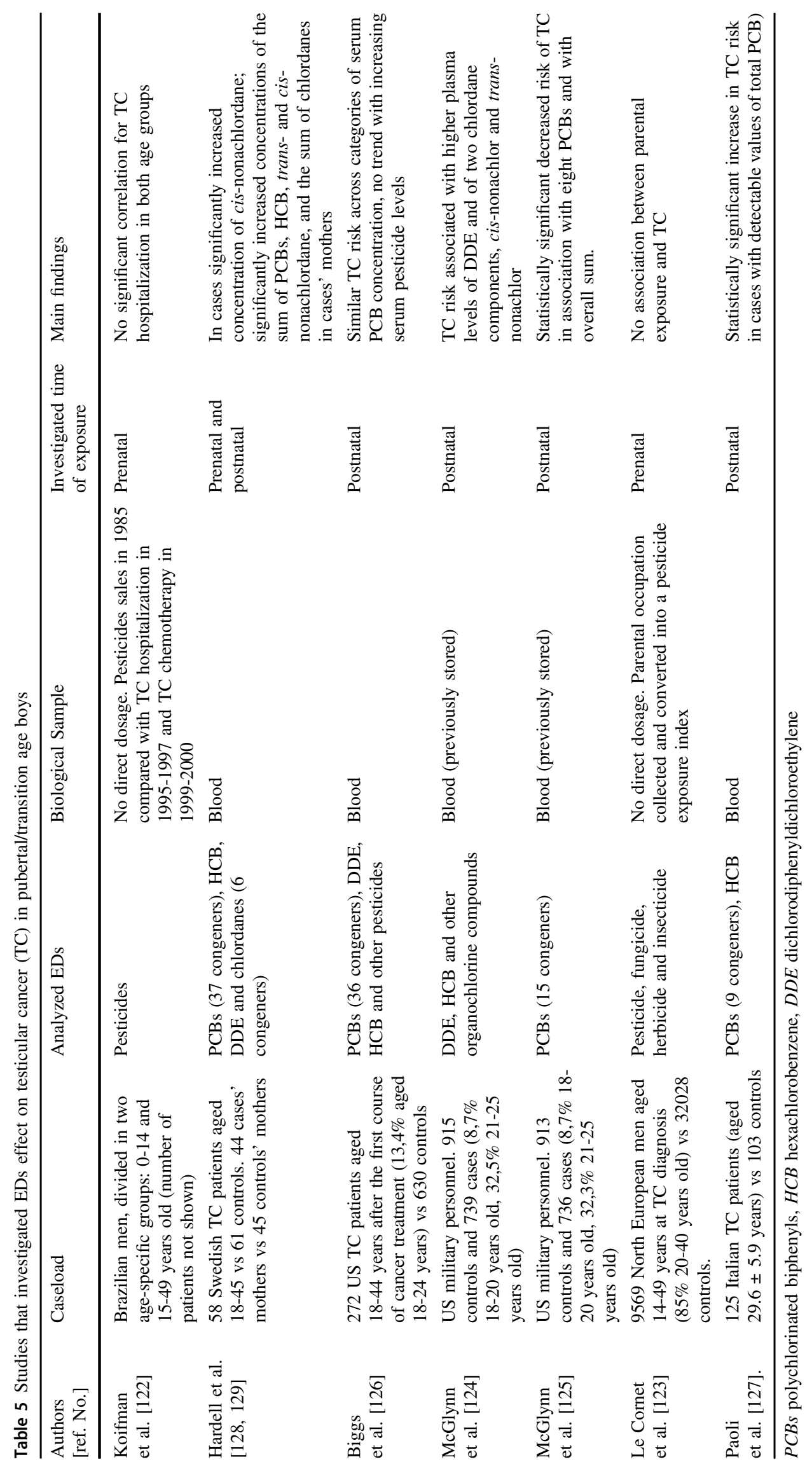

